\title{
Tau-Collocation Approximation Approach for Solving First and Second Order Ordinary Differential Equations
}

\author{
James E. Mamadu1', Ignatius N. Njoseh² \\ ${ }^{1}$ Department of Mathematics, University of Ilorin, Ilorin, Nigeria \\ ${ }^{2}$ Department of Mathematics and Computer Science, Delta State University, Abraka, Nigeria \\ Email: mamaduebimene@hotmail.com, njoseh@delsu.edu.ng
}

Received 25 January 2016; accepted 23 February 2016; published 26 February 2016

Copyright (C) 2016 by authors and Scientific Research Publishing Inc.

This work is licensed under the Creative Commons Attribution International License (CC BY). http://creativecommons.org/licenses/by/4.0/

c) (i) Open Access

\section{Abstract}

This paper presents Tau-collocation approximation approach for solving first and second orders ordinary differential equations. We use the method in the stimulation of numerical techniques for the approximate solution of linear initial value problems (IVP) in first and second order ordinary differential equations. The resulting numerical evidences show the method is adequate and effective.

\section{Keywords}

Ordinary Differential Equation (ODE), Initial Value Problem (IVP), Canonical Polynomial, Collocation

\section{Introduction}

The subject of Ordinary Differential Equation (ODE) is an important aspect of mathematics. It is useful in modeling a wide variety of physical phenomena-chemical reactions, satellite orbit, electrical networks, and so on. In many cases, the independent variable represents time so that the differential equation describes changes, with respect to time, in the system being modeled. The solution of the equation will be a representation of the state of the system. Consequently, the problem of finding the solution of a differential equation plays a significant role in scientific research, particularly, in the stimulation of physical phenomena. However, it is usually impossible to obtain direct solution of differential equations for systems to be modeled, especially complex ones encountered in real world problems. Since most of these equations are, or can be approximated by ordinary differential equations, a fast, accurate and efficient ODE solver is much needed. The Tau method was introduced by [1] to 
provide approximate polynomial solution for linear ordinary differential equation with polynomial coefficient.

The method takes advantage of the special properties of Chebychev polynomials. The main idea is to obtain an approximate solution of a given problem by solving an approximate problem. To further enhance the desired simplicity Lanczos introduced the systematic use of the canonical polynomials in the Tau method. The difficulties presented by the construction of such polynomials limited its application to first order ODE with the polynomial coefficient. The said difficulties were resolved by [2] when he proposed the generation of these canonical polynomials recursively. The beauty of the result of Ortiz is that the elements of canonical polynomials sequences by means of a simple recursive relation which is self starting and explicit. There are many literature developed concerning the Tau/Tau-collocation approximation method (see ([3]-[8])).

In this paper, we apply the Tau-collocation approximation method for the solution of linear initial value problems of the first and second order ODE in its differential and canonical form. We perform some numerical stimulation on some selected problems and compare the performance/effectiveness of the method with the analytic solutions given.

\section{The Tau Method}

Lanczos [1] approximated a solution $y(x)$ of the differential

$$
\sum_{r=o}^{m} P_{r}(x) y^{(r)}=f(x), 0 \leq x \leq 1
$$

where $P_{r}(x), f(x)$ are polynomials. $y^{(r)}(x)$ denotes the $r$ th order derivative of $y(x)$ with respect to $x$ and $y^{(0)}(x)$ taken simply as $y(x)$ by a polynomial

$$
y_{n}(x)=\sum_{r=o}^{n} a_{r} x^{r}, n<\infty
$$

and determines the coefficient $a_{r}, r=0(1) n$ of (2) such that $y_{n}(x)$ satisfies (1) perturbed by a term(s), which are calculated as part of the process. That is, $y_{n}(x)$ satisfies

$$
\sum_{r=0}^{m} P_{r}(x) y^{(r)}=f(x)+H_{n}(x) .
$$

Then

$$
H_{n}(x)=\sum_{r=o}^{m+s-1}{ }_{m+s-r} T_{n-m+r+1}^{*}, 0 \leq x \leq 1
$$

where $m$ is the order of the differential equation, $s$ is the number of over-determination,

$r=1(1) m+s$, are the parameters to be determined, and

$$
T_{r}^{*}(x)=\operatorname{Cos}\left[r \operatorname{Cos}^{-1}\left(\frac{2 x-a-b}{b-a}\right)\right] \equiv \sum_{k=0}^{r} C_{k}^{(r)} x^{k}
$$

is the $r$ th degree shifted Chebychev polynomial valid in the interval $[a, b]$ (assuming (1) is defined in this interval).

The free parameters in Equation (4) and the coefficient $a_{r}, r \in 0(1) n$ in (2) are obtained by equating the values of $x$ in (3) together with (1) to zero.

\subsection{Description of the Differential Form}

Considering the $m$ th order linear differential Equation ([1] [2])

$$
\begin{gathered}
L(y(x)):=\sum_{r=o}^{m} P_{r}(x) y^{(r)}=f(x), \\
y(0)=\alpha_{0}, y^{\prime}(0)=\alpha_{1}, \cdots, y^{(m-1)}(0)=\alpha_{(m-1)},
\end{gathered}
$$


with $y(x)$ as the exact solution in $a \leq x \leq b,|a|<\infty,|b|<\infty$.

We seek an approximate solution of the differential solution by the Tau method using the $n$th degree polynomial function

$$
y_{n}(x)=\sum_{r=0}^{m} P_{r}(x) x^{r}, n<\infty
$$

which satisfies the perturbed problem

$$
L(y(x)):=\sum_{r=0}^{m} P_{r}(x) y^{(r)}=f(x)+H_{n}(x)
$$

We equate the corresponding coefficient of $x$ in (8) and using the initial conditions

$$
y(0)=\alpha_{0}, y^{\prime}(0)=\alpha_{1}, \cdots, y^{(m-1)}(0)=\alpha_{(m-1)},
$$

We then solve the system of equation by Gaussian elimination method.

\subsection{Collocation Approach to the Tau Method}

The Lanczos Tau method in [4] is an economization process for a function that is implicitly defined by differential equation. Let us assume an approximation of the power series expansion $u(x)$ as

$$
u_{n}(x)=\sum_{r=o}^{n} a_{r}(x) x^{r}
$$

Consider an approximation to the residual $R_{n}(x)$ as

$$
R_{n}(x)={ }_{1} T_{n}^{*}(x)+{ }_{2} T_{n-1}^{*}(x)+\cdots+{ }_{m} T_{n-m+1}^{*}(x)
$$

Then by the Tau method, if

$$
L(u(x))=f(x)
$$

we have

$$
L(u(x))=f(x)+R_{n}(x)=f(x)+{ }_{1} T_{n}^{*}(x)+{ }_{2} T_{n-1}^{*}(x)+\cdots+{ }_{m} T_{n-m+1}^{*}(x)
$$

where $L$ is a linear differential operator of order $n$.

We collocate (12) at $x_{k}=k h, k=1(1) N$, where $h=\frac{b-a}{n+1}$ to have

$$
L\left(u\left(x_{k}\right)\right)=f\left(x_{k}\right)+R_{n}\left(x_{k}\right)=f\left(x_{k}\right)+{ }_{1} T_{n}^{*}\left(x_{k}\right)+{ }_{2} T_{n-1}^{*}\left(x_{k}\right)+\cdots+{ }_{m} T_{n-m+1}^{*}\left(x_{k}\right)
$$

The parameter $1,2, \cdots, m$ may be eliminated leaving the unknown coefficient $a_{k}, k=0(1) n$ with $(n+1)$ linear equations which can be solved by Gaussian elimination.

\section{Error Estimation}

Let us in this section consider and obtain the error estimator for the approximate solution of (1) and (9). Let $E_{n}=u(x)-u_{n}(x)$ be the error function of $u_{n}(x)$ to $u(x)$, where $u(x)$ is the exact solution of (1) and (9). Therefore $u_{n}(x)$ satisfies the perturbed problems:

$$
L\left(u_{n}(x)\right):=\sum_{r=0}^{m} P_{r}(x)\left(u_{n}(x)\right)^{(r)}=f(x)+H_{n}(x), x \in[a, b],|a|<\infty,|b|<\infty, n<\infty
$$

and

$$
L\left(u_{n}(x)\right):=\sum_{r=0}^{m} a_{r} x^{r}=f(x)+H_{n}(x)
$$


where $H_{n}$ is uniquely defined as in (4).

To obtain the perturbation term $H_{n}(x)$, we substitute the computed solution $u_{n}(x)$ such that

$$
L\left(u_{n}(x)\right):=\sum_{r=0}^{m} P_{r}(x)\left(u_{n}(x)\right)^{(r)}-f(x)=H_{n}(x)
$$

and

$$
L\left(u_{n}(x)\right):=\sum_{r=0}^{m} a_{r} x^{r}-f(x)=H_{n}(x)
$$

We then proceed to find an approximate $E_{n, N}(x)$ to the error function $E_{n}(x)$ in the same manner as we did for the solution of (1) and (9).

Thus, the error function, $E_{n}$, satisfy the problem

$$
L\left(E_{n}\right):=\sum_{r=0}^{m} P_{r}(x) E_{n}^{r}-f(x)=-H_{n}(x)
$$

and

$$
L\left(E_{n}\right):=\sum_{r=0}^{m} a_{r} x^{r}-f(x)=0
$$

which satisfies the conditions prescribed.

\section{Illustrative Examples}

In this section, two initial value problems are considered to show the efficiency of the method.

\section{Example 1}

Consider linear initial value problem in second order ordinary differential equation

$$
\left\{\begin{array}{l}
u^{\prime \prime}(x)+u(x)=0 \\
u(0)=1, u^{\prime}(1)=3
\end{array}\right.
$$

We solve [4] for $n=2$ using; (i) The Tau method; and (ii) Tau-collocation method.

The analytic solution is $u(x)=\operatorname{Cos} x-\frac{\operatorname{Sin} x(\operatorname{Cos} 1-3)}{\operatorname{Sin} 1}$.

By the Tau method we obtain the linear differential operator as

$$
L \equiv\left(\frac{\mathrm{d}^{2}}{\mathrm{~d} x^{2}}+1\right)
$$

The associated canonical polynomials are obtained as follows:

$$
\begin{gathered}
L x^{r}=\left(\frac{\mathrm{d}^{2}}{\mathrm{~d} x^{2}}+1\right) x^{r} \\
L x^{r}=r(r-1) L Q_{r-2}(x)+L Q_{r}(x) \\
L^{-1} \cdot L x^{r}=L^{-1} \cdot L\left[r(r-1) Q_{r-2}(x)+Q_{r}(x)\right] \\
\Rightarrow x^{r}=r(r-1) Q_{r-2}(x)+Q_{r}(x) \\
\Rightarrow Q_{r}(x)=x^{r}-r(r-1) Q_{r-2}(x)
\end{gathered}
$$

The canonical polynomials, $Q_{r}(x), r \geq 0$, obtained here can easily be obtained from [3] where the generalized form of the canonical polynomials was reported.

For $r=0(1) 2$, we have 


$$
Q_{0}(x)=1, Q_{1}(x)=x \text { and } Q_{2}(x)=x^{2}-2
$$

These polynomials are substituted into Equation (12) to give

$$
L u_{n}(x)={ }_{1} T_{n}^{*}(x)+{ }_{2} T_{n-1}^{*}(x)
$$

Using Equation (5),

$$
\begin{gathered}
L u_{n}(x)={ }_{1} \sum_{r=0}^{n} C_{r}^{(n)} x^{r}+{ }_{2} \sum_{r=0}^{n-1} C_{r}^{(n-1)} x^{r}(x) \\
L u_{n}(x)={ }_{1} \sum_{r=0}^{n} C_{r}^{(n)} L Q_{r}(x)+{ }_{2} \sum_{r=0}^{n-1} C_{r}^{(n-1)} L Q_{r}(x) \\
u_{n}(x)={ }_{1} \sum_{r=0}^{n} C_{r}^{(n)} Q_{r}(x)+{ }_{2} \sum_{r=0}^{n-1} C_{r}^{(n-1)} Q_{r}(x)
\end{gathered}
$$

Since $n=2$

$$
u_{2}(x)={ }_{1} \sum_{r=0}^{2} C_{r}^{(2)} Q_{r}(x)+{ }_{2} \sum_{r=0}^{1} C_{r}^{(n-1)} Q_{r}(x)
$$

Now,

$$
\begin{gathered}
T_{0}(x)=1, T_{1}(x)=2 x-1 \text { and } T_{2}(x)=8 x^{2}-8 x+1 \\
u_{2}(x)={ }_{1}\left(C_{0}^{(2)} Q_{0}(x)+C_{1}^{(2)} Q_{1}(x)+C_{2}^{(2)} Q_{2}(x)\right)+{ }_{2}\left(C_{0}^{(1)} Q_{0}(x)+C_{1}^{(1)} Q_{1}(x)\right) \\
\Rightarrow u_{2}(x)={ }_{1}\left(1-8 x+8\left(x^{2}-2\right)\right)+{ }_{2}(-1+2 x)
\end{gathered}
$$

Using initial conditions on Equation (23) and simplifying further we get the approximate solution as

$$
u_{2}(x)=1+\frac{46}{15} x-\frac{16}{15} x^{2}
$$

Considering the Tau-collocation method we have:

Let

$$
\begin{gathered}
u_{2}(x)=\sum_{r=0}^{2} a_{r}(x) x^{r} \\
u_{2}(x)=a_{0}+a_{1} x+a_{2} x^{2} \\
u_{2}^{\prime}(x)=a_{1}+2 a_{2} x \\
u_{2}^{\prime \prime}(x)=2 a_{2}
\end{gathered}
$$

Substituting into (13) we have,

$$
\begin{gathered}
2 a_{2}+a_{0}+a_{1} x+a_{2} x^{2}={ }_{1} T_{2}^{*}(x)+{ }_{2} T_{1}^{*}(x) \\
2 a_{2}+a_{0}+a_{1} x+a_{2} x^{2}={ }_{1}\left(1-8 x+8\left(x^{2}-2\right)\right)+{ }_{2}(-1+2 x)
\end{gathered}
$$

Now collocating at $x_{k}=k h, h=\frac{b-a}{n+1}, k=1,2,3$ and using the initial conditions, we obtain the approximate solution as

$$
u_{2}(x)=1+\frac{46}{15} x-\frac{16}{15} x^{2}
$$

\section{Example 2}

Consider the first order IVP 


$$
L y(x)=(1+x) y^{\prime}(x)+y(x)=0, y(0)=1
$$

The exact solution is $y(x)=\frac{1}{x+1}$

For the given IVP, we can deduce that $m=1$ and $s=0$.

The differential formulation is as follows:

Let

$$
y_{n}(x)=\sum_{r=o}^{n} a_{r} x^{r}
$$

Taking $n=5$

$$
\begin{gathered}
L y_{5}(x)=\sum_{r=o}^{m+s-1}{ }_{m+s-r} T_{n-m+r+1}^{*}(x) \\
\Rightarrow L y_{5}(x)=\sum_{r=o}^{0}{ }_{1} T_{5}^{*}
\end{gathered}
$$

where

$$
T_{5}^{*}=\sum_{r=o}^{5} C_{r}^{(5)} X^{r}
$$

hence

$$
(1+x) y_{5}^{\prime}(x)+y_{5}(x)={ }_{1} T_{5}^{*}(x)
$$

but

$$
L y_{5}(x)={ }_{1} \sum_{r=o}^{5} C_{r}^{(5)} x^{r}
$$

Using (28) and (30) in (29) we obtain,

$$
(x+1) \sum_{r=o}^{5} r a_{r} x^{r-1}+\sum_{r=o}^{5} r a_{r} x^{r}={ }_{1} \sum_{r=o}^{5} C_{r}^{(5)} x^{r}
$$

Expanding and equating coefficients of powers of $x$, the resulting linear equations together with the equations obtained using the initial conditions is written in the form,

$$
A \underline{x}=\underline{b}
$$

where

$$
\begin{gathered}
\left(\begin{array}{cccccccc}
1 & 1 & 0 & 0 & 0 & 0 & \cdots & C_{0}^{(5)} \\
0 & 2 & 2 & 0 & 0 & 0 & \cdots & C_{1}^{(5)} \\
0 & 0 & 3 & 3 & 0 & 0 & \cdots & C_{2}^{(5)} \\
0 & 0 & 0 & 4 & 4 & 0 & \cdots & C_{3}^{(5)} \\
0 & 0 & 0 & 0 & 5 & 5 & \cdots & C_{4}^{(5)} \\
0 & 0 & 0 & 0 & 0 & 0 & \cdots & C_{5}^{(5)} \\
1 & 0 & 0 & 0 & 0 & 0 & \cdots & 0
\end{array}\right) \\
\underline{x}=\left(a_{0}, a_{1}, a_{2}, a_{3}, a_{4}, a_{5}, 1\right)^{\mathrm{T}}, \\
\underline{b}=(0,0,0,0,0,0,1)^{\mathrm{T}}
\end{gathered}
$$

Using Equation (5), we obtain the following values, 


$$
C_{0}^{(5)}=-1, C_{1}^{(5)}=50, C_{2}^{(5)}=-400, C_{3}^{(5)}=1120, C_{4}^{(5)}=-1280 \text { and } C_{5}^{(5)}=512 .
$$

Using these values in the matrix and solving by Gaussian elimination method, we have,

$$
a_{0}=1, a_{1}=-\frac{2339}{2342}, a_{2}=\frac{1132}{1171}, a_{3}=-\frac{932}{1171}, a_{4}=\frac{512}{1171}, a_{5}=-\frac{128}{1171},{ }_{1}=\frac{3}{2342}
$$

The approximate solution is:

$$
y_{5}(x)=\frac{1}{2342}\left(2342-2339 x+2264 x^{2}-1864 x^{3}+1024 x^{4}-256 x^{5}\right)
$$

\section{Discussion of Results}

The results obtained above show that the Tau-collocation method is appropriate for the solution of linear initial value problems of first and second kind ordinary differential equations. From the tables (Table 1 and Table 2) of results presented above, we observe that the approximate solution considered at grid points, $n=2$ and $n=5$, for examples 1 and 2 converges to the analytic solution with maximum absolute errors of $10^{-3}$ and $10^{-5}$ respectively. We obtain satisfactory results because of the excellent convergence rate of the Tau-colloca-

Table 1. Numerical results for example 1.

\begin{tabular}{|c|c|c|c|}
\hline$X$ & Exact Solution & Approximate solution, $n=2$ & Error \\
\hline 0.1 & 0.9090909 & 0.9090418 & $4.19133 \mathrm{e}-05$ \\
\hline 0.2 & 0.8333333 & 0.8332214 & $1.1195 \mathrm{e}-04$ \\
\hline 0.3 & 0.7692308 & 0.7691735 & $5.7276 \mathrm{e}-05$ \\
\hline 0.4 & 0.7142857 & 0.7143198 & $3.4081 \mathrm{e}-05$ \\
\hline 0.5 & 0.6666667 & 0.6667378 & $7.1164 \mathrm{e}-05$ \\
\hline 0.6 & 0.6250000 & 0.6250298 & $2.9821 \mathrm{e}-05$ \\
\hline 0.7 & 0.5882353 & 0.5881915 & $4.3800 \mathrm{e}-05$ \\
\hline 0.8 & 0.5555556 & 0.5554809 & $7.4633 \mathrm{e}^{-}-05$ \\
\hline 0.9 & 1.5263158 & 0.5265873 & $2.8445 \mathrm{e}^{-}-05$ \\
\hline 0.10 & 0.5000000 & 0.5000000 & $0.0000 \mathrm{e}+00$ \\
\hline
\end{tabular}

\begin{tabular}{cccc}
\hline$X$ & Exact Solution & Approximate solution, $n=2$ & Error \\
\hline 0.1 & 1.2868265 & 1.2960000 & $9.1735 \mathrm{e}-03$ \\
0.2 & 1.5607954 & 1.5706667 & $9.8712 \mathrm{e}-03$ \\
0.3 & 1.8191694 & 1.8240000 & $4.8306 \mathrm{e}-03$ \\
0.4 & 2.0593669 & 2.0560000 & $3.3669 \mathrm{e}-03$ \\
0.5 & 2.2789879 & 2.2666667 & $1.2321 \mathrm{e}-02$ \\
0.6 & 2.4758379 & 2.4560000 & $1.9838 \mathrm{e}-02$ \\
0.7 & 2.6479502 & 2.6240000 & $2.3950 \mathrm{e}-02$ \\
0.8 & 2.7936051 & 2.7706667 & $2.2938 \mathrm{e}-02$ \\
0.9 & 2.9113471 & 2.8960000 & $1.5347 \mathrm{e}-02$ \\
1.0 & 3.0000000 & 3.0000000 & $0.0000 \mathrm{e}+00$ \\
\hline
\end{tabular}

Table 2. Numerical results for Example 2. 
tion approximation method, which is very close to the minimax polynomial which minimizes the maximum error in approximation. Thus, the approximate solution will match the analytic solution as $\mathrm{n}$ increases.

\section{Conclusions}

This paper has considered Tau-collocation approximation approach for solving particular first and second order ordinary differential equations. The method offers several advantages which include, among others:

1) It takes advantages of the special properties of Chebychev polynomials which can be easily generated recursively;

2) Elements of canonical polynomials sequences by means of a simple re-cursive relation which is self starting and explicit; and

3) It can easily be programmed for experimentation.

Tau-Collocation method can be extended to higher order ordinary differential equations and stochastic differential equations. It can also be used to solve integro-differential and stochastic integro-differential equations.

\section{References}

[1] Lanczos, C. (1956) Applied Analysis. Prentice-Hall, Engle-Wood Cliffs, New Jersey.

[2] Ortiz, E.L. (1969) The Tau Method. SIAM Journal on Numerical Analysis, 6, 480-492. http://dx.doi.org/10.1137/0706044

[3] Yisa, B.M. and Adeniyi, R.B. (2012) A Generalized Formulation for Canonical Polynomials for M-Th Order Non Over-Determined Ordinary Differential Equations. Internal Journal of Engineering Research and Technology. www.ijert.org

[4] Coleman, J.P. (1974) Lanczos Tau Method. IMA Journal of Applied Mathematics, 7, 85-97.

[5] El-Daou, M.K., Ortiz, E.L. and Samara, H. (1993) A Unified Approach to the Tau Method and Chebychev Series Expansion Technique. Computer and Mathematics with Applications, 25, 73-82. http://dx.doi.org/10.1016/0898-1221(93)90145-L

[6] Ortiz, E.L. (1975) Step by Step Tau-Method—Part 1. Computer and Mathematics with Applications, 1, 381-392. http://dx.doi.org/10.1016/0898-1221(75)90040-1

[7] Khajah, H. (1999) Tau-Method Approximation of a Generalized Epstein-Hubbel Elliptic Type Integral. Computer and Mathematics, 68, 1615-1621. http://dx.doi.org/10.1090/S0025-5718-99-01128-X

[8] Sam, C.N. (2004) Numerical Solution of Partial Differential Equations with the Tau-Collocation Method. M. Phil Thesis, City University of Hong Kong. http://dspace.cityu.edu.hk/handle/2031/4614 